\title{
Air-Assisted Atomization of Beveled Needle Point Injector
}

\author{
M.S. Raza ${ }^{1}$, A. Valiaev², K.A. Sallam ${ }^{1 *}$ \\ ${ }^{1}$ Mechanical and Aerospace Engineering, Oklahoma State University, Tulsa, Oklahoma \\ ${ }^{2}$ Technology Development Center, Oklahoma State University, Stillwater, Oklahoma \\ ${ }^{*}$ Corresponding author email : khaled.sallam@okstate.edu
}

\begin{abstract}
The air-assisted atomization of a micro liquid jet injected from beveled needle point style injector is studied experimentally motivated by its potential applications in biomedical devices. The primary breakup mechanism is investigated using doubled pulsed shadowgraphy. The results include the intact core length, the location of the onset of liquid jet breakup, size of drops at onset. The results are interpreted using phenomenological analysis, and relevance of the findings to biomedical applications are discussed.
\end{abstract}

\section{Keywords}

primary breakup, instability, injector geometry, micro jet.

\section{Introduction}

Air assisted atomizers has wide spread applications including atomization of biomedical suspensions. This atomization technique has been extensively studied using numerical simulations [1-3] and experimental approaches [4-6]. It could successfully be combined with other atomization techniques. Ahmed et al., reported a new design of a hybrid air-blast and electrostatic atomizer that can be useful for the atomization of highly viscous fuels like biodiesel [5] and demonstrated that droplet and ligament size are reduced with increasing of spray charge, with the maximum effect of the charge being found for the lowest Weber number [5]. Funada et al., provided a comprehensive theoretical model of temporal instability of a circular liquid jet in a coflowing high speed air stream using viscous potential flow [1]. The model predicts that with increases in air stream speed there is a reduction of breakup fragments of liquid [1]. The geometry of the injector received considerable interest, e.g. Glathe et al., experimentally studied the effect of air-assisted atomization of liquid in the setup where liquid was delivered through a central tube, and the air was delivered through the annular orifice on the outer radius of the liquid tube. The authors demonstrated noticeable effects of eccentricity of the central tube (i.e., that changed the geometry of the air co-flow relative to the liquid stream) on the air-assisted atomization using high speed airflow [4]. A study by Kumar et al., [7] found substantial contribution of the liquid-centered coaxial injector's exit geometry on liquid jet breakup in a high-speed annular air stream. By employing a swirl air blast atomizer design, Soni et.al., demonstrated that the use of annular swirl air can effectively reduce the jet breakup length, and contribute to better air-fuel mixing and vaporization characteristics [8]. Yang et.al., have developed a model for the explicit expression of Sauter Mean Diameter (SMD) which favorably matched the experimental data [9]. According to findings by Marmottant et al. [10], an air-assisted atomization is initialized by a Kelvin-Helmholtz instability which generates interfacial waves on the liquid core by aerodynamic forces. This first stage is followed by the surface perturbations due to the Rayleigh-Taylor instability, where liquid structures protruding from the liquid surface are accelerated by aerodynamic interactions, leading to disintegration of the intact jet core [10]. The above two-stages process is referred to as primary atomization which, in cases where droplets exceed a critical size, is followed by secondary atomization to form smaller droplets. According to this model of high-velocity air 
stream atomization developed by Yang et al., the liquid jet is first subjected to the KelvinHelmholtz instability which forms a liquid ligament followed by airflow assisted breakup of the filament into fine droplets through the effects of Rayleigh-Taylor instability [9]. Zhang et al. has developed a model for air-assisted atomization of high-viscous glycerol/water jet injected into a coaxial airflow where the atomization occurs at elevated ambient pressures [11]. Most of these studies however, were limited to relatively large injectors. Of interest in the present study is the impact of shrinking injector size on the breakup outcomes. The objective of this paper is to investigate the breakup outcomes of a beveled micro needle, used to inject test liquid into a coflowing airstream injected from a larger beveled needle motivated by the potential usage as disposable and inexpensive atomizers in variety of medical and industrial applications.

\section{Material and Methods}

The present coflowing atomizer is assembled from two beveled needles (16G and 30G one inside the other, with the smaller needle resting against the inner wall of the larger needle, as shown in Fig. 1. The pressure of city water line (50 psig) was used to feed the test liquid (water). Shop air (maximum 110 psig) air was used to generate the coflowing air in the outer needle, as shown in Fig. 1. An inline filter (not shown in Fig. 2) is used to clean the water before deliverying it to the needle to avoid blockages. Two different Rotameters (Omega Engineering \& Dakota Instruments) are used to measure the flow rate of air and water that is supplied to the atomizer.

The diagnostics consists of double-pulsed shadowgraphy. Two Nd:YAG lasers (New wave laser, $70 \mathrm{~mJ} /$ pulse, $10 \mathrm{~Hz}$ ) generated the laser beams. The two beams were aligned, expanded and collimated as shown in Fig. 2. A diffusing screen were used to provide the background illumination. A double exposure camera (pco.2000, 2048 × 2048 pixel) with 60 $\mathrm{mm}$ Nikkor lens was sued to record the image. A delay generator is used to time the two laser pulses and the camera exposures. Two images are taken with the desired pulse separation for velocity measurements. An oscilloscope is used to measure the pulse separation. The test conditions include 5 different water flow rates. At each water flow rate three different air velocities are tested to study the effects of air on the atomizer behavior. ImageJ software was used to analyze the images. The liquid jet velocity was calculated by dividing the liquid volumetric flow rate by the internal cross section area of the inner needle. The gas (air) velocity was calculated by dividing the volumetric flow rate of the air flow, as measured by the rotameter, by the cross section area of the annular flow between the outer and inner needles. The results include onset of droplets, and intact breakup length.

\section{Results and Discussion}

The flow visualization of the liquid jet without coflowing air is shown in Fig. 3. The was done by carrying the liquid flow rate and using only the inner needle. The results show beveled needle injector behave similar to cylindrical injector with Rayleigh breakup at low flow rates. The effect of coflowing air was investigated by varying the flow rate as shown in Fig. 4 . The air and liquid flow rates are controlled independently in the present setup. The air velocity does not affect the liquid flow rate, as shown in Fig. 5. This allowed flexibility in the setting up the test matrix.

The breakup length of the liquid jet (defined as the length of the intact core length) is controlled by controlling the flowrate of both liquid and air flowrates, as shown in Fig. 6 . The droplet sizes at onset was measured and plotted as function of the air velocity, as shown in Fig. 7. The drop sizes are reduced as the air velocity is increased. 
Of interest is the spatial distribution of spray and the effects of secondary breakup. Due to the high magnification of the present setup the depth of focus was small. This is become problematic as the measuring volume is moved downstream and very few droplets are in focus with many droplets out of focus do the three dimensional nature of the spray. In order to measure the droplets sizes as function of distance from the jet exit it is crucial to use three dimensional imaging technique. Therefore the next step it to use digital holography. Additionally the air velocity currently is calculated assuming constant density between the rotameter and the needle exit. This will be corrected in future work. The research team plan to generate a breakup regime map and to investigate the spray size distribution as function of distance from the injector exit. and large data set of drop sizes and drop velocities.

\section{Conclusions}

The present study investigated experimentally the outcome of a micro coflowing injector assembled form off the shelf beveled needles. The results show that this simple design would provide a disposable and economical coflowing injector capable of atomization of micro liquid jets. The droplets sizes and the breakup length can be controlled through the liquid and gas flow rates. The generated droplet sizes (60 microns) are unfortunately lager than what typical medical sprays (e.g for nasal delivery) should be (typically 10 micros). A further study to reduce the generated study is planned in the near future.

\section{Acknowledgments}

The assistance in designing and assembling the present injector by the New Product Development Center, Oklahoma State University, under the leadership of Dr. Robert Taylor, is gratefully acknowledged.

\section{Nomenclature}

ALR Air to liquid Ratio [-]

$L F R \quad$ Liquid Flow Rate [mlpm]

We Weber number [-]

\section{References}

[1] Funada, T., Joseph, D. D., Saitoh, M., and Yamashita, S., 2006, "Liquid jet in a high Mach number air stream," International Journal of Multiphase Flow, 32, pp. 20-50.

[2] Eggers, J., and Villermaux, E., 2008, "Physics of liquid jets," Reports on Progress in Physics, 71.

[3] Kozul, M., Costa, P. S., Dawson, J. R., and Brandt, L., 2020, "Aerodynamically driven rupture of a liquid film by turbulent shear flow," Physical Review Fluids, 5(12), pp. 1-27.

[4] Glathe, A., Wozniak, G., and Richter, T., 2001, "The Influence of Eccentricity on the Performance of a Coaxial Prefilming Air-Assist Atomizer," Atomization and Sprays, 11, pp. 2133.

[5] Ahmed, T., Kourmatzis, A., and Masri, A., 2021, "Atomization behaviour of a hybrid airblast-electrostatic atomizer for spray combustion," Fuel, 288, pp. 1-13.

[6] Murugan, R., Balusamy, S., and Kolhe, P., 2020, "Experimental Study of!Liquid Spray Mode of!Twin Fluid Atomizer Using Optical Diagnostic Tool," Flow, Turbulence and Combustion, 106, pp. 261-289.

[7] Kumar, A., and Sahu, S., 2020, "Influence of nozzle geometry on primary and large-scale instabilities in coaxial injectors," Chemical Engineering Science, 221.

[8] Soni, S. K., and Kolhe, P., 2020, "Liquid jet breakup and spray formation with annular swirl air," International Journal of Multiphase Flow, 134.

[9] Yang, L., Gao, Y., Li, J., and Fu, Q., 2020, "Theoretical atomization model of a coaxial gasliquid jet," Physics of Fluids, 32(124108). 
[10] Marmottant, P., and Villermaux, E., 2004, "On spray formation," Journal of Fluid Mechanics, 498, pp. 73-111.

[11] Zhang, F., Zirwes, T., Müller, T., Wachter, S., Jakobs, T., Habisreuther, P., Zarzalis, N., Trimis, D., and Kolb, T., 2020, "Effect of elevated pressure on air-assisted primary atomization of coaxial liquid jets: Basic research for entrained flow gasification," Renewable and Sustainable Energy Reviews, 134, pp. 1-11. 


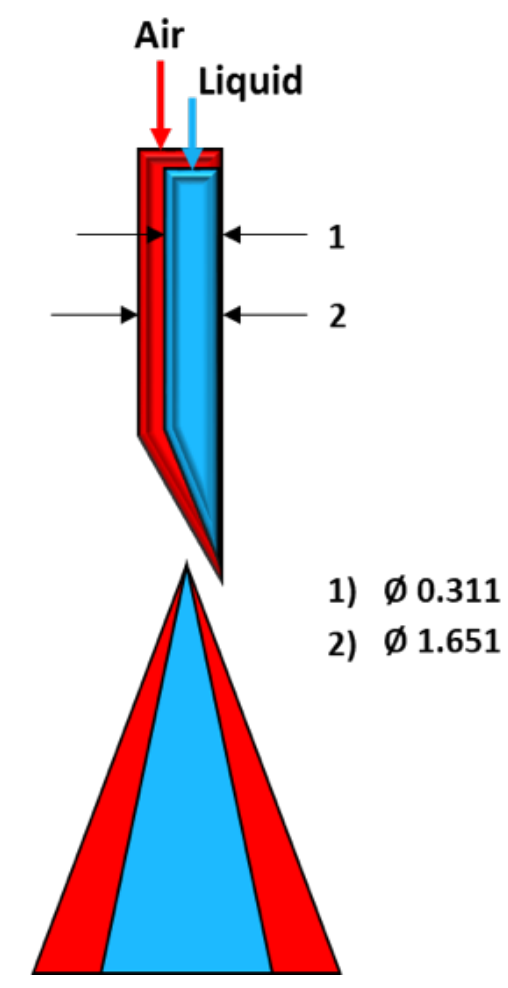

Figure 1. Sketch of the present coflow injector.
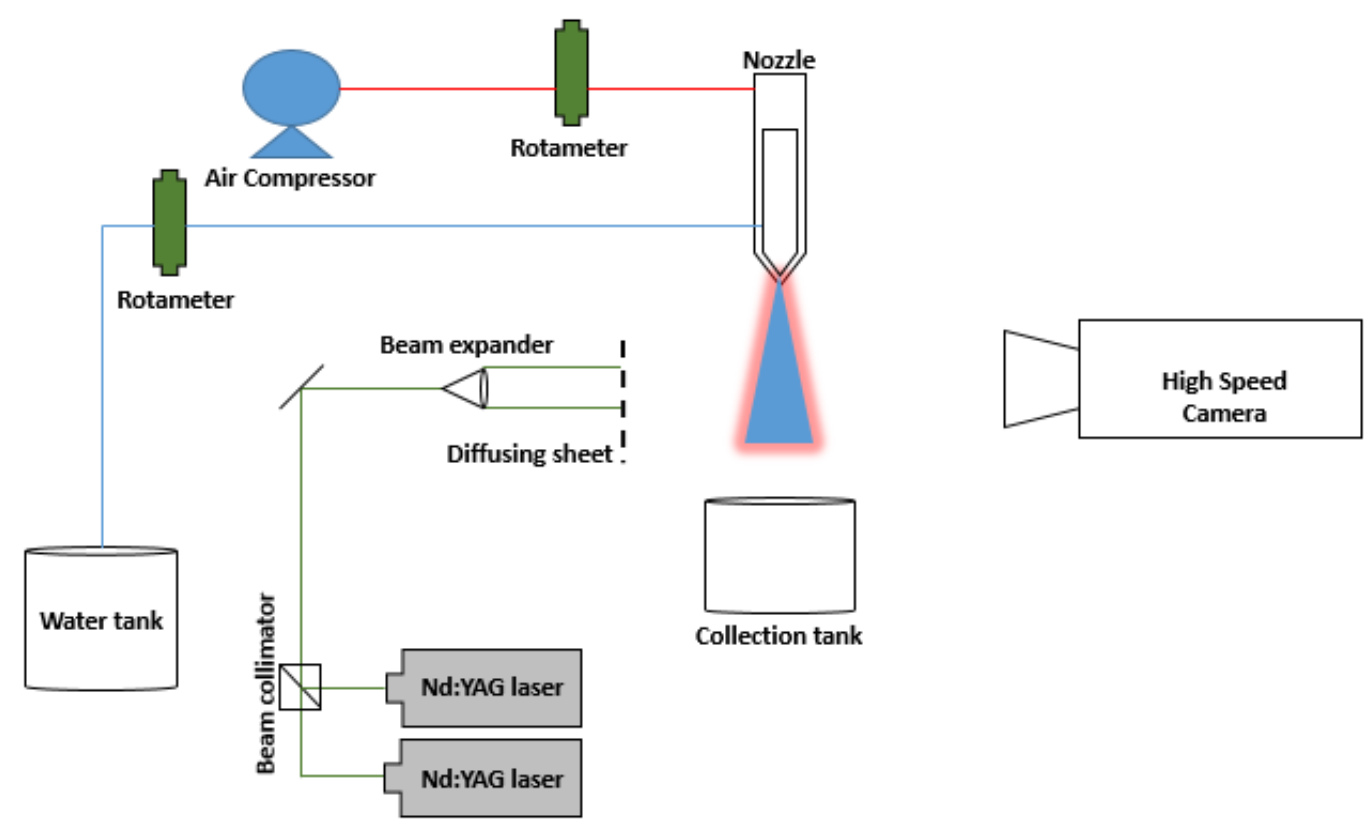

Figure 2. Sketch of the apparatus. 


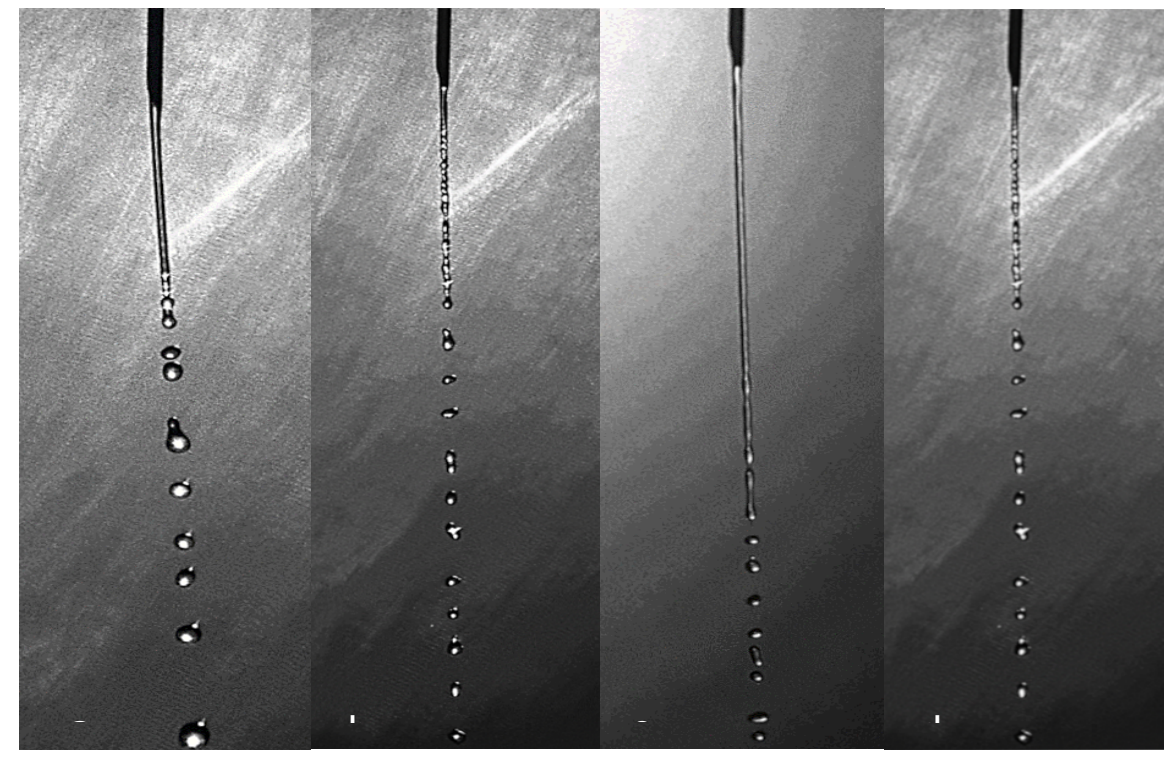

Figure 3. Liquid jet in still air at four different liquid flow rates in gallons per day. a) 0.4 , b) 0.9 , c) 1.5 and d) 3.7 .

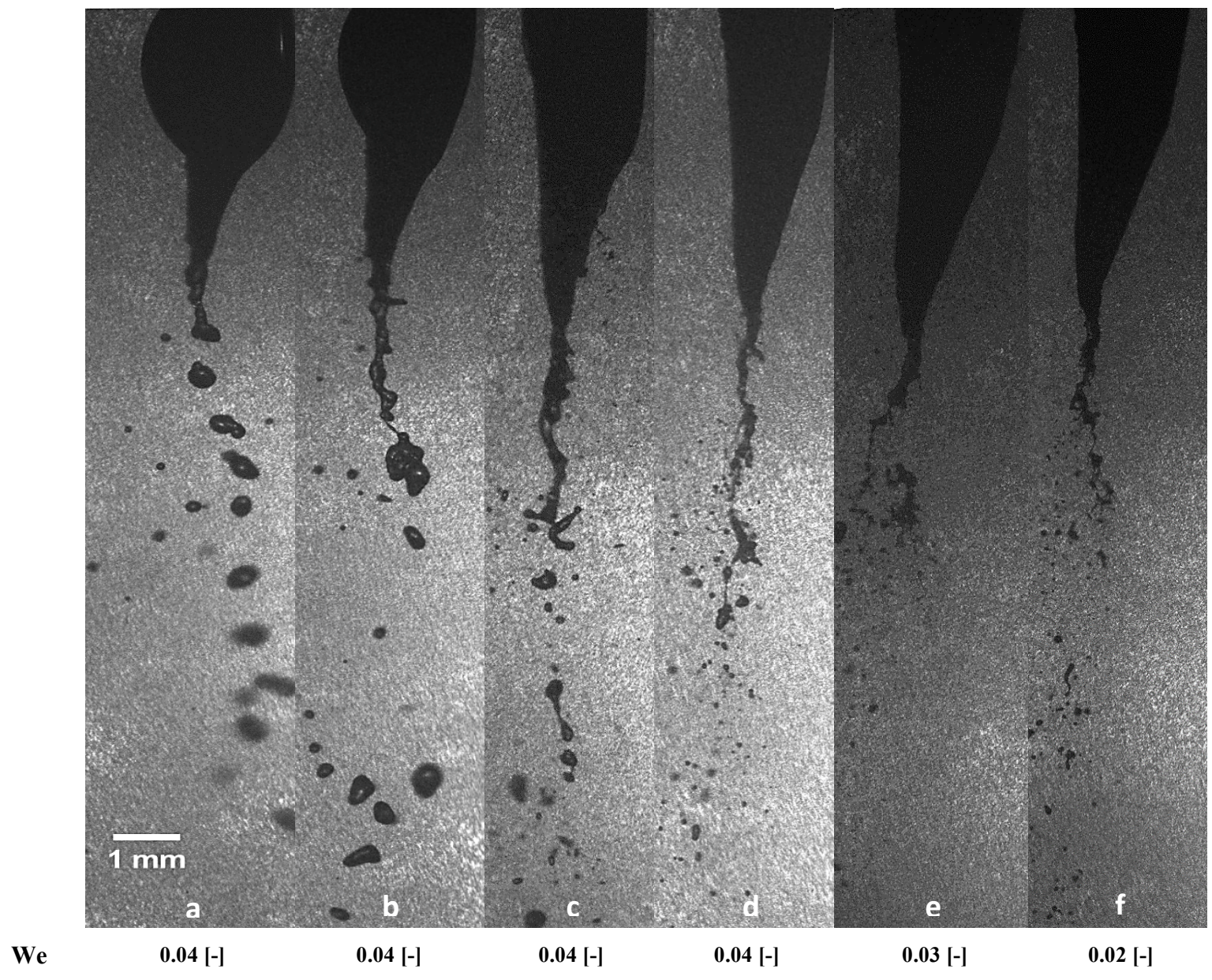

Figure 4. The effect of air flow rate on the breakup length. 


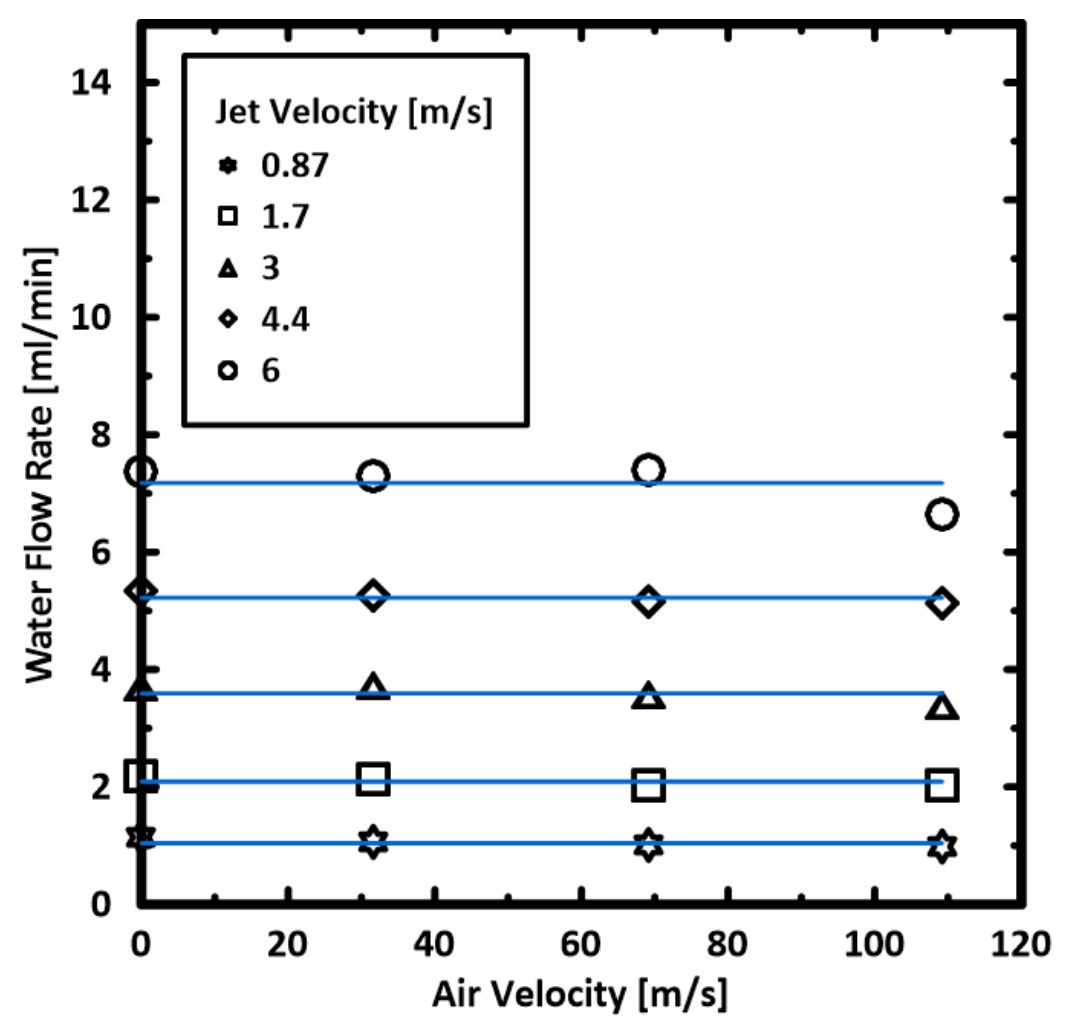

Figure 5. Liquid flow rate as a function of Air Velocity, tested at 5 different water flow rates and three different air velocities.

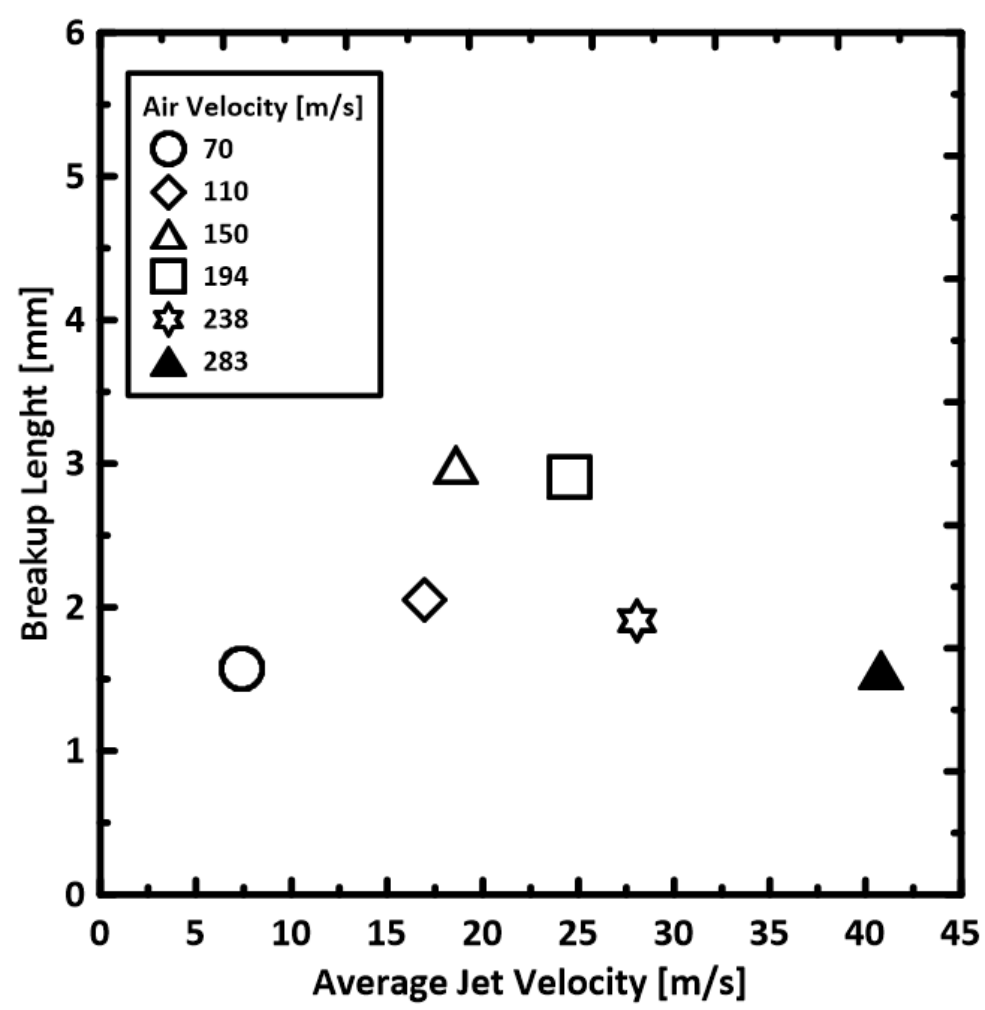

Figure 6. The breakup length of the jet as a function of the jet velocity based on 6 different air flow rates. 


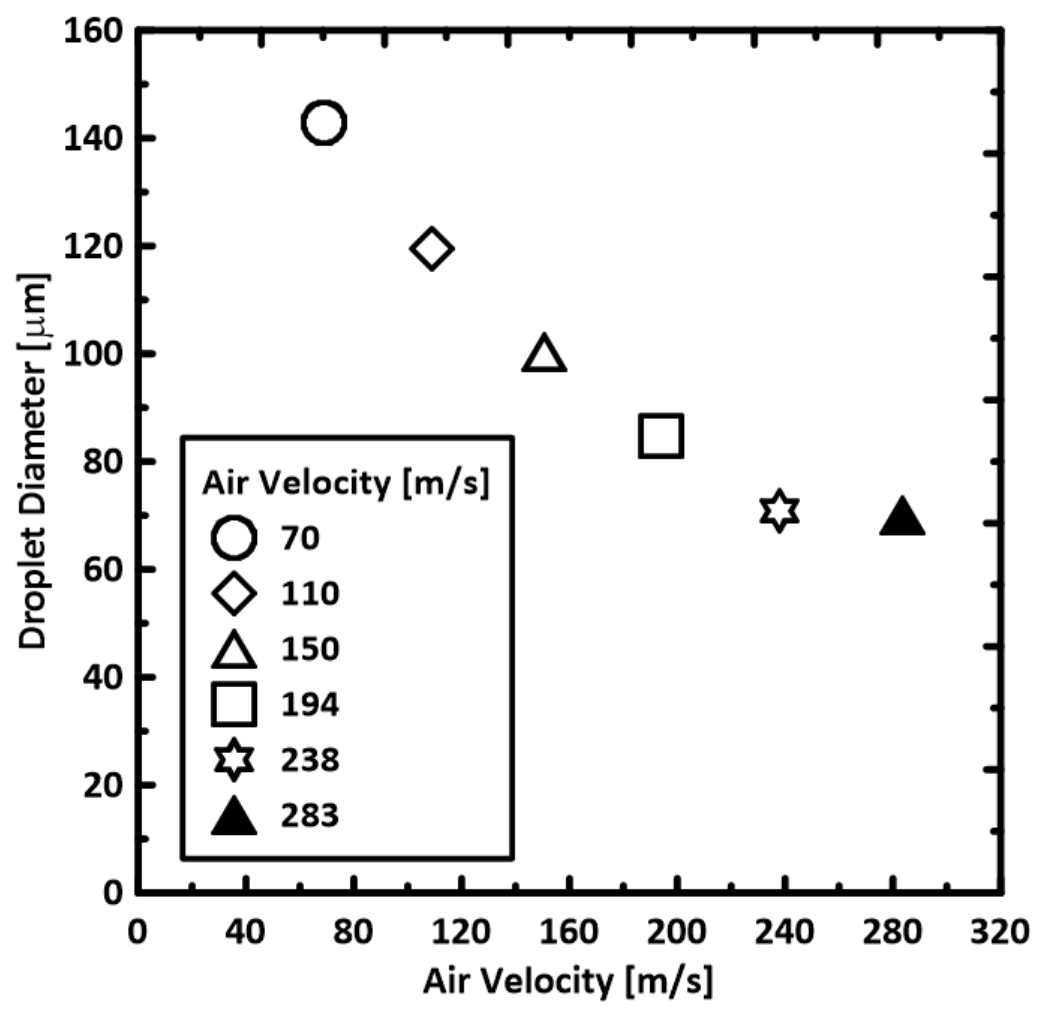

Figure 7. Droplet diameter as function of Air velocity tested at 6 different Air flow rates. 